\title{
Cantilever Magnetometry of Individual Ni Nanotubes
}

\author{
D. P. Weber, ${ }^{\dagger}$ D. Rüffer, ${ }^{\ddagger}$ A. Buchter, ${ }^{\dagger}$ F. Xue, ${ }^{\dagger}$ E. Russo-Averchi, ${ }^{\ddagger}$ R. Huber, ${ }^{\S}$ P. Berberich, ${ }^{\S}{ }^{\ddagger}$. Arbiol, ${ }^{\#}$ \\ A. Fontcuberta i Morral, ${ }^{\ddagger}$ D. Grundler, ${ }^{\S}, \|$ and M. Poggio $*, \dagger$ \\ ${ }^{\dagger}$ Departement Physik, Universität Basel, 4056 Basel, Switzerland \\ ${ }^{\ddagger}$ Laboratoire des Matériaux Semiconducteurs, Institut des Matériaux, Ecole Polytechnique Fédérale de Lausanne, 1015 Lausanne, \\ Switzerland \\ ${ }^{\S}$ Lehrstuhl für Physik funktionaler Schichtsysteme, Physik Department E10, Technische Universität München, James-Franck-Str. 1, \\ 85748 Garching, Germany \\ "Faculté des Sciences et Techniques de l'Ingénieur STI, Ecole Polytechnique Fédérale de Lausanne, 1015 Lausanne, Switzerland \\ \#Institució Catalana de Recerca i Estudis Avançats and Institut de Ciència de Materials de Barcelona, 08193 Bellaterra, CAT, Spain
}

\section{Supporting Information}

\begin{abstract}
Recent experimental and theoretical work has focused on ferromagnetic nanotubes due to their potential applications as magnetic sensors or as elements in high-density magnetic memory. The possible presence of magnetic vortex states - states which produce no stray fieldsmakes these structures particularly promising as storage devices. Here we investigate the behavior of the magnetization states in individual $\mathrm{Ni}$ nanotubes by sensitive cantilever magnetometry. Magnetometry measurements are carried out in the three major orientations, revealing the presence of different stable magnetic states. The observed behavior is well-described by a model based on the presence of uniform states at high applied magnetic fields and a circumferential onion state at low applied fields.
\end{abstract}
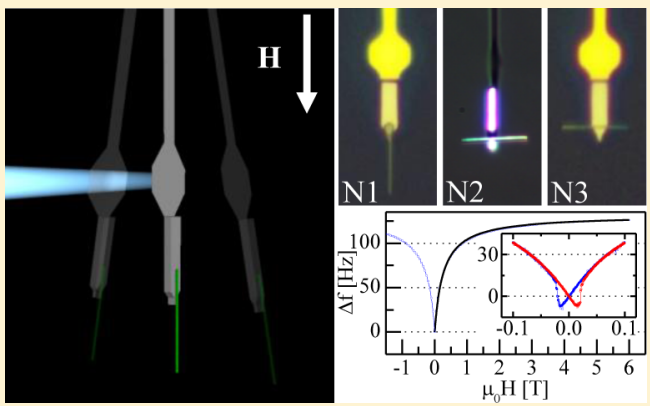

KEYWORDS: Magnetic nanotubes, cantilever magnetometry, magnetic tubular architectures, nanomagnetic states

$\mathrm{T}$ he synthesis and investigation of ferromagnetic nanostructures has been motivated both by a large number of potential applications and by fundamental questions about the physics of nanometer-scale magnetism. Magnetic nanoparticles have potential biological and biomedical applications, ${ }^{1-6}$ applications in high-resolution magnetic imaging, ${ }^{7-9}$ as magnetic sensors, ${ }^{10}$ and as dense magnetic storage media. ${ }^{11}$ At the same time, the low-dimensionality of these structures results in magnetic configurations not present in macroscopic magnets. ${ }^{12-15}$ In particular, magnetic nanotubes distinguish themselves from magnetic nanowires in that they support corefree magnetic states. Such configurations avoid the magnetic point singularity along the axis of the structure, ${ }^{16}$ thereby resulting in a fast and controllable reversal process. ${ }^{17}$ In addition, previously unforeseen dynamic effects are possible in nanotubes. Domain walls moving in nanotubes are predicted to avoid a Walker breakdown and give rise to Cherenkov-like spin wave emission. ${ }^{18}$ Both numerical simulations ${ }^{19}$ and analytical calculations $^{20,21}$ show that the tubular geometry favors two main in-plane states: a uniform axial state (UAS) with the magnetic moments pointing along the tube axis and a global vortex state (GVS) with moments pointing circumferentially around the tube. Due to their flux-closure configuration, vortex states produce much lower stray fields than uniform states; as a result, magneto-static interactions between nanomagnets could be reduced resulting in densely packed magnetic memories. Further possibilities include a multidomain state $(\mathrm{MDS})^{17}$ composed of a mixture of uniform and vortex domains, an onion state $(\mathrm{OS})^{22,23}$ consisting of two oppositely oriented circumferential domains, and uniform states in which all magnetic moments align along the applied field. For nanotubes with tailored magneto-crystalline or interfacial anisotropy a radial out-of-plane state (ROS), in which magnetic moments align along the tube radius, is also possible. Here we present experimental measurements of individual $\mathrm{Ni}$ nanotubes supporting the presence of various states including uniform states, the MDS, and the OS.

We use sensitive dynamic-mode cantilever magnetometry ${ }^{24}$ to investigate the magnetic states of the nanotubes. Our approach allows us to measure the moment, anisotropy, and switching behavior of a single $\mathrm{Ni}$ nanotube as a function of applied magnetic field and orientation. Until recently, magnetization measurements had only been carried out on large ensembles of ferromagnetic nanotubes. ${ }^{25-31}$ Due to the distribution in size and orientation, these measurements are difficult to interpret. In 2012, Rüffer et al. probed the magnetic states of a single Ni nanotube in transport measurements using the anisotropic magnetoresistance effect. ${ }^{23}$ Here we use a different method to measure the magnetization and effective

Received: August 8, 2012

Revised: October 19, 2012

Published: November 6, 2012 

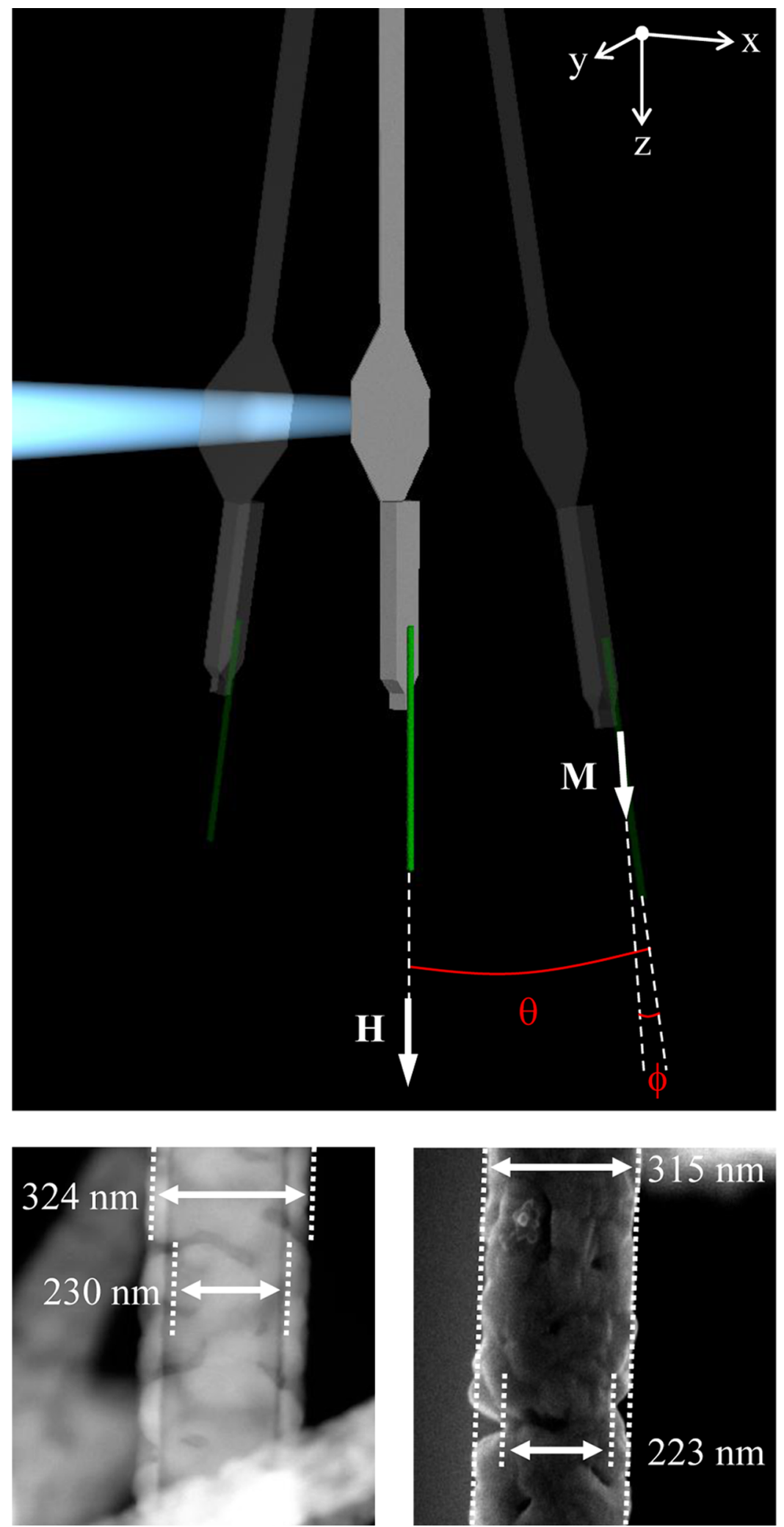

Figure 1. Top: Schematic diagram showing the oscillating cantilever (gray), laser light from the interferometer (white), the Ni nanotube (green), and the relative orientations of the cantilever axis, the applied magnetic field $\mathbf{H}$, and the Ni nanotube magnetization $\mathbf{M}$. Bottom: Transmission electron micrograph (TEM) (left), and scanning electron micrograph (SEM) (right) of a single Ni nanotube. Arrows indicate both the maximal inner and the outer diameter of the Ni shell.

magnetic anisotropy of individual $\mathrm{Ni}$ nanotubes, shedding further light on their magnetic states. Due to its high sensitivity, cantilever magnetometry is well-suited for the detection of the weak magnetic response of a variety of nanometer-scale systems. We note recent measurements of the persistent currents in normal metal rings, ${ }^{32}$ of the magnetization of 

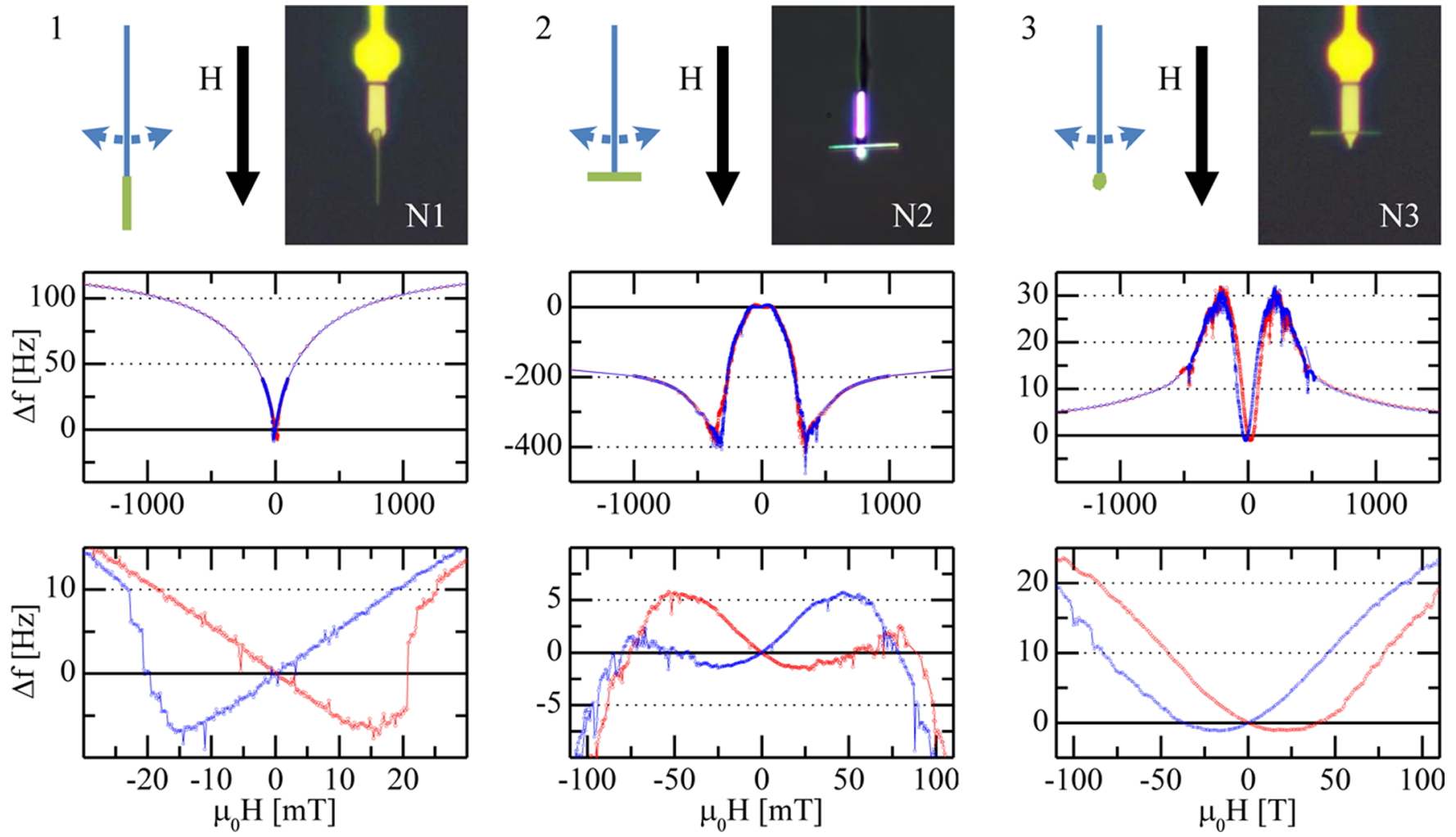

Figure 2. Cantilever magnetometry measurements in three major orientations. Each column shows measurements from one of the major orientations as indicated by the schematic diagrams at the top; from left to right we show configurations 1,2 , and 3 , with optical micrographs of the nanotube samples N1, N2, and N3. The lower two rows show the corresponding measurements of $\Delta f$ as a function of $H$ in different field ranges for each configuration. Red (blue) points represent data taken while sweeping $H$ in the positive (negative) direction.

superconducting nanostructures, ${ }^{33}$ and of magnetization reversal in a single iron-filled carbon nanotube ${ }^{34}$ and a single Ni nanorod. ${ }^{35}$

Cantilever magnetometry experiments are carried out in a vacuum chamber with a pressure below $1 \times 10^{-6} \mathrm{mbar}$ at the bottom of a ${ }^{4} \mathrm{He}$ cryostat. A superconducting magnet allows the application of an external magnetic field $\mu_{0} \mathrm{H}$ of up to $6 \mathrm{~T}$ along the cantilever axis $\hat{z}$. Each single $\mathrm{Ni}$ nanotube that we investigate is affixed to the tip of an ultrasoft cantilever (see Supporting Information, Figures S1 and S2) with less than 100 fL of epoxy (Gatan G1) applied under an optical microscope by means of precision micromanipulators (Narishige MMO202ND). The nanomagnets are produced by atomic layer deposition (ALD) of $\mathrm{Ni}$ on a nanowire template made of $\mathrm{GaAs}^{23}$ (see Figure 1). Since the GaAs nanowires have the shape of a slightly sloped truncated cone, the $20-\mu \mathrm{m}$-long nanotubes have an outer diameter which narrows from around $360 \mathrm{~nm}$ at one end to $280 \mathrm{~nm}$ at the other. The thickness of the $\mathrm{Ni}$ shell is just over $40 \mathrm{~nm}$ (see Supporting Information, Table $\mathrm{S} 1$ ). The single-crystal Si cantilevers used here are $150 \mu \mathrm{m}$ long, $4 \mu \mathrm{m}$ wide, and $0.1 \mu \mathrm{m}$ thick and include a $18-\mu \mathrm{m}$-long, $1-\mu \mathrm{m}$ thick mass on their end. ${ }^{36}$ The motion of the levers is detected using laser light focused onto a $12-\mu \mathrm{m}$-wide paddle near the mass-loaded end and reflected back into an optical fiber interferometer. ${ }^{37} 100 \mathrm{nW}$ of light are incident on the paddle from a temperature-tuned $1550 \mathrm{~nm}$ distributed feedback laser diode. At $\mathrm{T}=4.2 \mathrm{~K}$ and $\mu_{0} \mathrm{H}=0 \mathrm{~T}$, the nanotube-loaded cantilevers have resonant frequencies $f_{0}=\omega_{0} /(2 \pi)$ between 2 and $3 \mathrm{kHz}$ and intrinsic quality factors around $Q_{0}=3 \times 10^{4}$. Their spring constants $k_{0}$ are determined to be close to $60 \mu \mathrm{N} /$ $\mathrm{m}$ through measurements of thermal noise spectra at several different temperatures (see Supporting Information, Table S1). The interferometric cantilever deflection signal is fed through a field programmable gate array (FPGA) circuit (National Instruments) back to a piezoelectric element which is mechanically coupled to the cantilever. In this way, we are able to self-oscillate the cantilever at its fundamental resonance frequency and at a desired amplitude. Self-oscillation allows for fast and accurate measurement of the cantilever resonance frequency.

We measure $f_{0}$ as a function of $H$ at $T=4.2 \mathrm{~K}$ with a selfoscillation amplitude of $x_{\text {rms }}=40 \mathrm{~nm}$. For such small cantilever deflections $x \ll l_{\mathrm{e}}$, where $l_{\mathrm{e}}=105 \mu \mathrm{m}$ is the effective cantilever length for the fundamental mode, the $\mathrm{Ni}$ nanotube tilts by an angle $\theta=x / l_{\mathrm{e}}$ with respect to $\hat{z}$ as shown in Figure 1 . The measured shift in resonance frequency $\Delta f$ depends on the torque acting between the $\mathrm{Ni}$ nanotube and $H$. The experiments are carried out for identically grown Ni nanotubes mounted on the cantilever tip in the three major orientations. Configuration 1 corresponds to the nanotube's symmetry axis $\hat{z}^{\prime}$ aligned along $\hat{z}$. Configurations 2 and 3 correspond to $\hat{z}^{\prime}$ aligned along $\hat{x}$ and $\hat{y}$, respectively, where $\hat{x}$ corresponds to the direction of cantilever deflection. The orientations, samples, and $\Delta f$ as a function of $H$ are shown in Figure 2. Note that the three configurations are realized using three different nanotubes fabricated in the same growth and ALD process; we label the nanotubes $\mathrm{N} 1, \mathrm{~N} 2$, and $\mathrm{N} 3$, respectively.

The dependence of $\Delta f$ on $H$ is fundamentally different for each configuration. In configuration $1, \Delta f$ is positive for large $|H|$ and approaches a constant value. At low fields, the data show a clear hysteresis with switching occurring through a series of discrete steps in $\Delta f$. In the other configurations the 

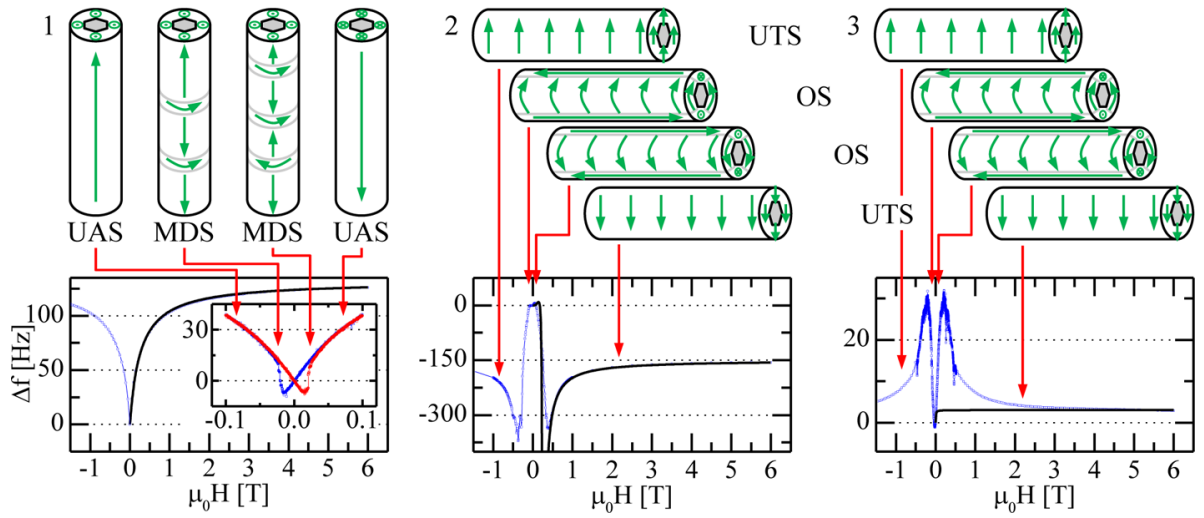

Figure 3. Magnetic state progression and model fits in three major orientations. In the top row we show schematic diagrams of the magnetization states described in the text. The lower row shows measurements of $\Delta f$ (blue points), and the fit functions based on eq 2 (black lines) as a function of $H$ for each configuration. Red arrows indicate magnetic fields corresponding to the specified state.

dependence is more complex: in configuration $2, \Delta f$ becomes negative, and for large $|H|$ eventually approaches a constant negative value; minima in $\Delta f$ are observed near +400 and -400 $\mathrm{mT}$. In configuration $3, \Delta f$ is positive and goes through a maximum, and for large $|H|$ approaches a small positive value. Both configurations 2 and 3 show hysteresis at low fields. For all orientations, we measure a negligible dependence of the mechanical dissipation on $H$ beyond that intrinsic to the $\mathrm{Si}$ cantilevers. $^{38}$ The fluctuation-dissipation theorem implies that magnetic-field dependent dissipation is the result of magnetic moment fluctuations in the sample or the cantilever. The lack of additional magnetic fluctuations due to the Ni nanotubes is likely due to their large magnetic anisotropy.

To interpret our data we begin by making the simplifying assumption that our nanotube behaves as a single-domain magnetic particle, that is, its magnetization is uniform and rotates in unison. For high enough applied fields, the nanotube is magnetized to saturation, and thus this single-domain assumption is valid. We therefore describe the nanotube's magnetic state by the orientation of its total magnetization vector M. More complex states deviating from this assumption will be addressed separately later. Since the Ni nanotube is polycrystalline and does not exhibit magneto-crystalline anisotropy, we assume the nanotube to exhibit only shape anisotropy. The total energy of the system can be written as the sum of the cantilever energy, the Zeeman energy, and an effective anisotropy energy: $:^{39}$

$$
E=\frac{1}{2} k_{0}\left(l_{\mathrm{e}} \theta\right)^{2}-M V H \cos (\theta-\phi)+K V \sin ^{2} \phi
$$

where $V$ is the volume of the nanotube, $K$ is its anisotropy in the plane of the cantilever oscillation, and $\phi$ is the angle between $\mathbf{M}$ and $\hat{z}^{\prime}$. To calculate $\phi$, we minimize the energy of the system with respect to this angle. The solutions must satisfy both $\partial E / \partial \phi=0$ and $\partial^{2} E / \partial \phi^{2}>0$. Although solutions for $\phi$ are difficult to obtain exactly, since $\theta \ll 1$, we can expand $\phi$ as a function of $\theta$ to first order around $\theta=0$. We then substitute the expansion for $\phi(\theta)$ into the expression for the torque acting on the cantilever, $\tau=-\partial E / \partial \theta=-k_{0} l_{\mathrm{e}}^{2} \theta-H M V \sin (\theta-\phi)$. Keeping only terms up to first order in $\theta$ and approximating the cantilever as a simple harmonic oscillator, we solve for the cantilever's frequency shift $\Delta f=f-f_{0}$, where $f$ is the measured resonance frequency and $f_{0}$ is the resonance frequency at $H=$ 0 . The expected frequency shift as a function of $H$ is (see Supporting Information for full derivation):

$$
\Delta f=\left\{\begin{array}{cc}
\frac{\omega_{0}}{4 \pi k_{0} l_{\mathrm{e}}^{2}}\left(\frac{2 H K V}{H+\frac{2 K}{M}}\right) & H>-\frac{2 K}{M} \\
\frac{\omega_{0}}{4 \pi k_{0} l_{\mathrm{e}}^{2}}\left(\frac{2 H K V}{H-\frac{2 K}{M}}\right) & H<\frac{2 K}{M} \\
\frac{\omega_{0}}{4 \pi k_{0} l_{\mathrm{e}}^{2}}\left[\frac{H^{2} M^{2} V}{2 K}\right. & K<0 \text { and }|H| \\
\left(\frac{2 H^{2}-\left(\frac{2 K}{M}\right)^{2}}{M} \mid\right. & <\left|\frac{2 K}{M}\right|
\end{array}\right.
$$

Singularities at $H= \pm(2 K / M)$ reflect the breakdown of the small angle approximation, and the solutions become invalid near this field. The first two solutions correspond to $\mathbf{M}$ pointing along $\pm \hat{z}$ respectively. The third solution, valid only for $K<0$, corresponds to $\mathbf{M}$ along an easy axis perpendicular to $\hat{z}$ (the implication of a negative $K$ ) and rotating toward $\hat{z}$ with increasing $H$.

Using this model based on a single-domain magnetic particle, we can fit the data taken in configuration 1 . The data and the fit function, given by eq 2 , are plotted together in Figure 3. $\omega_{0}, k_{0}$, and $V$ are set to their measured values (see Supporting Information, Table S1), while $M=M_{S}=330 \pm 50 \mathrm{kA} / \mathrm{m}$ and $K$ $=44 \pm 6 \mathrm{~kJ} / \mathrm{m}^{3}$ are extracted as fit parameters for sample N1. Here the effective anisotropy $K$ represents the anisotropy of the easy axis oriented along the nanotube's axis of symmetry $\hat{z}^{\prime}$ in the plane of the cantilever oscillation.

While at high fields $\left(\mu_{0} \mathrm{H}>100 \mathrm{mT}\right)$, the measurements are consistent with a UAS, at low fields the step-like structures shown in Figure 2 (see also Supporting Information, Figure S3) cannot be described by the uniform magnetization model. These discrete magnetization steps indicate the presence of transition states between the two UASs. In addition, the number of steps, which occur at slightly different fields each time the field is swept, suggest the presence of three to five MDSs. According to calculations, ${ }^{17}$ MDSs are possible and are configured as depicted in Figure 3; that is, they consist of uniform axially saturated domains separated by azimuthal, or vortex-like, domain walls. 
A similar fit using eq 2 can be made for the data taken in configuration 2 as shown in Figure 3. Here $K<0$ since $\mathbf{H}$ is directed along a hard axis of the nanotube, and the cantilever oscillates in a plane defined by this hard axis and its easy axis $\hat{z}^{\prime}$. In this orientation and at sufficiently high field, the magnetic moments in the nanotube will align uniformly along the applied field, forming a uniform transverse state (UTS). The magnetometry measurement should therefore result in an $M$ equal to that measured in configuration 1 and a $K$ with an equal magnitude and the opposite sign. In fact, we extract $M=M_{S}=$ $420 \pm 90 \mathrm{kA} / \mathrm{m}$ and $K=-52 \pm 11 \mathrm{~kJ} / \mathrm{m}^{3}$ as fit parameters for sample N2. These values are equal to the values extracted in configuration 1 for $\mathrm{N} 1$ within the error of the measurement, which is dominated by the difficulty of determining each nanotube's exact volume. Although eq 2 describes the data for large $|H|$, the measurements deviate from the model at low fields. In particular, for $\left|\mu_{0} H\right|<100 \mathrm{mT}$ the data show a clear hysteresis. According to eq 2, only one stable solution of $\Delta f$ exists for $K<0$, unlike in the case of $K>0$ where two exist for $|H|<(2 K / M)$. With only one stable solution, hysteretic behavior cannot be reproduced; therefore we conclude that the description of a single uniform magnetization in the nanotube breaks down at low applied fields. Furthermore, the low-field hysteresis points to the presence of a magnetization state with positive effective anisotropy for small $H$.

One explanation for the differing behavior at high and low fields is that, while at high fields the Ni nanotube is uniformly magnetized, at low fields a more complex state emerges. One possible state, which has been predicted to be stable for such samples at low fields, is the OS. ${ }^{23}$ This state is shown schematically in Figure 3 and consists of azimuthally oriented magnetization domains separated by axially oriented domain walls. The OS has a total magnetization $M<(1 / 2 \pi) \int_{0}^{\pi} 2 M_{S}$ $\sin \theta^{\prime} \mathrm{d} \theta^{\prime}=(2 / \pi) M_{S}$ due to the azimuthal orientation of its domains and a positive effective anisotropy, related to the energy required to rotate the azimuthally oriented magnetization domains toward the nanotube axis. The presence of the OS at low fields could explain the hysteresis observed in configuration 2. Due to its lower magneto-static energy compared to saturated states, the OS is favored in low magnetic fields. For this reason we suppose the Ni nanotube to undergo a transition from the OS to the UTS as a function of increasing $|H|$. Given the region of deviation between the simple model and the data, this transition region is likely to be between $\left|\mu_{0} H\right|=0$ and $2 \mathrm{~T}$. Here we hypothesize the presence of a MDS with some segments of the nanotube in the OS and some in the UTS.

The aforementioned model is also consistent with the data measured in configuration 3 on sample N3. The high field behavior is well-described by a UTS with $M=M_{S}=375 \mathrm{kA} / \mathrm{m}$ and a small positive magnetic anisotropy $K=0.90 \pm 0.25 \mathrm{~kJ} /$ $\mathrm{m}^{3}$. Note that we choose $M_{S}$ of $\mathrm{N} 3$ to be between the values extracted for $\mathrm{N} 1$ and $\mathrm{N} 2$, since the high field behavior of the fit in configuration 3 is highly insensitive to $M$. $\mathbf{H}$ is directed along a hard axis of the nanotube, and the cantilever oscillates in a plane perpendicular to its axis of symmetry $\hat{z}^{\prime}$. For an ideal nanotube in this orientation, no anisotropy should be present due to its circular symmetry; because of inevitable imperfections of real $\mathrm{Ni}$ nanotubes (see Figure 1), this symmetry is broken, and therefore we measure a small, in this case positive, $K$. For small $|H|$ the data deviate from this small positive anisotropy behavior, showing the presence of an unsaturated low-field state as observed in configuration 2. Hysteresis again appears for $\left|\mu_{0} H\right|<100 \mathrm{mT}$, and a transition region exists for $\left|\mu_{0} H\right|<2 \mathrm{~T}$. In this case, the low-field magnetometry points to a state with a larger positive anisotropy in this plane than the UTS. Once again, this low-field behavior is consistent with the OS. In this plane the OS has a positive effective anisotropy, related to the energy required to move the axially oriented domain walls and thus rotate the magnetization around the nanotube axis. The total magnetization is $M<(2 / \pi) M_{S}$ due to the azimuthal orientation of its domains. Frequency measurements in both configurations 2 and 3 show pronounced and reproducible structures as a function of $H$ for $\left|\mu_{0} H\right|<1 \mathrm{~T}$. These changes in $\Delta f$, and thus in magnetic torque, likely result from the gradual transition of the low-field OS to the UTS throughout the volume of the nanotube.

A GVS, which has a total magnetization $M=0$, should appear in our cantilever magnetometry measurements as a range in $H$ for which $\Delta f=0$. The ROS, which also has a total magnetization $M=0$ and would produce $\Delta f=0$, cannot be achieved since the $\mathrm{Ni}$ nanotubes are composed of an isotropic ferromagnet without crystalline anisotropy. As long as the GVS is stable for a significant range, that is, a range greater than 10 $\mathrm{mT}$, it would be observable in our experiment. In Figures 2.2 and 2.3 for $\mu_{0}|H| \approx 50 \mathrm{mT}, \Delta f=0$ for a small field range. While this behavior is consistent with the GVS, we cannot exclude that $\Delta f=0$ might be produced by a MDS with $M=0$ or with the appropriate combination of magnetization $M$ and anisotropy $K$. In minor hysteresis loops of the cantilever magnetometry (see Supporting Information, Figure S4), we can produce states with $\Delta f=0$ for field ranges of up to $50 \mathrm{mT}$. Again this evidence is consistent with the GVS but does not exclude the presence of other states. On the other hand, Rüffer et al. report evidence for a GVS in similar Ni nanotubes. ${ }^{23}$ The discrepancy may be due to differences in the geometrical parameters of the nanotubes, indicating what is already known from numerical and analytical calculations: the GVS is supported only for nanotubes which meet specific geometric conditions.

In conclusion we have presented experimental evidence for an onion and a multidomain state (OS, MDS) in ALD-grown $\mathrm{Ni}$ nanotubes. Dynamic cantilever magnetometry measurements of single nanotubes in the three principal orientations highlight the stability of complex low-field magnetic configurations. The characteristics of these low-field states are compatible with both the OS and the MDS as predicted by various theoretical works. From the cantilever magnetometry data above, we cannot unambiguously identify the global vortex state; a specific MDS may account for the same behavior. From measurements on different nanotubes, the developed analytical model provides us with consistent values for the saturation magnetization $M_{S}=375 \pm 70 \mathrm{kA} / \mathrm{m}$ and the anisotropy constant $|K|=48 \pm 9 \mathrm{~kJ} / \mathrm{m}^{3}$ for the easy axis. The $M_{S}$ measured in the Ni nanotubes is equal within the error to the value of 406 $\mathrm{kA} / \mathrm{m}$ known for bulk crystalline $\mathrm{Ni}$ at low temperature. ${ }^{40}$ Future high-resolution X-ray magnetic circular dichroism photoelectron emission microscopy (XMCD-PEEM) ${ }^{14,15}$ or magnetic force microscopy $(\mathrm{MFM})^{22,41}$ experiments on such magnetic nanotubes could provide further evidence for the presence of an OS or a GVS.

\section{ASSOCIATED CONTENT}

\section{S Supporting Information}

Full derivation of the model, video of the fabrication of the $\mathrm{Ni}$ nanotubes, SEMs of the measured nanotubes, study of the step- 
like structures of configuration 1 , minor hysteresis loops, and a table of sample and cantilever specifications. This material is available free of charge via the Internet at http://pubs.acs.org.

\section{AUTHOR INFORMATION}

\section{Corresponding Author}

*E-mail: martino.poggio@unibas.ch.

\section{Notes}

The authors declare no competing financial interest.

\section{ACKNOWLEDGMENTS}

The authors thank Prof. J. Arbiol from ICREA/ICMAB for making TEM images and Sascha Martin of the Mechanical Workshop at the Physics Department of the University of Basel for important contributions to the measurement apparatus. We acknowledge support from the Canton Aargau, the Swiss National Science Foundation (SNF, Grant No. 200020140478), the Swiss Nanoscience Institute, and the National Center of Competence in Research for Quantum Science and Technology. The research has also received funding from the European Community's Seventh Framework Programme (FP7/2007-2013) under Grant Agreement No. 228673, MAGNONICS.

\section{REFERENCES}

(1) Pankhurst, Q. A.; Connolly, J.; Jones, S. K.; Dobson, J. J. Phys. D: Appl. Phys. 2003, 36, R167.

(2) Son, S. J.; Reichel, J.; He, B.; Schuchman, M.; Lee, S. B. J. Am. Chem. Soc. 2005, 127, 7316.

(3) Leslie-Pelecky, D. L.; Labhasetwar, V. D.; Kraus, R. H., Jr. In Advanced Magnetic Nanostructures; Sellmyer, D., Skomski, R., Eds.; Springer: New York, 2006; pp 461-482.

(4) Lee, D.; Cohen, R. E.; Rubner, M. F. Langmuir 2007, 23, 123.

(5) Amstad, E.; Zurcher, S.; Wong, J. Y.; Textor, M.; Reimhult, E. Small 2009, 5, 1334.

(6) Kim, D.-H.; Rozhkova, E. A.; Ulasov, U. V.; Bader, S. D.; Rajh, T.; Lesniak, M. S.; Novosad, V. Nat. Mater. 2010, 9, 165.

(7) Khizroev, S.; Kryder, M. H.; Litvinov, D. Appl. Phys. Lett. 2002, 81, 2256.

(8) Poggio, M.; Degen, C. L. Nanotechnology 2010, 21, 342001.

(9) Campanella, H.; Jaafar, M.; Llobet, J.; Esteve, J.; Vázquez, M.; Asenjo, A.; del Real, R. P.; Plaza, J. A. Nanotechnology 2011, 22, 505301.

(10) Maqableh, M. M.; Huang, X.; Sung, S.-Y.; Reddy, K. S. M.; Norby, G.; Victora, R. H.; Stadler, B. J. H. Nano Lett. 2012, 12, 41024109.

(11) Parkin, S. S. P.; Hayashi, M.; Thomas, L. Science 2008, 320, 190.

(12) Wang, Z. K.; Kuok, M. H.; Ng, S. C.; Lockwood, D. J.; Cottam, M. G.; Nielsch, K.; Wehrspohn, R. B.; Gösele, U. Phys. Rev. Lett. 2002, 89, 027201.

(13) Topp, J.; Podbielski, J.; Heitmann, D.; Grundler, D. Phys. Rev. B 2008, 78, 024431 .

(14) Streubel, R.; Thurmer, J.; Makarov, D.; Kronast, F.; Kosub, T.; Kravchuk, V.; Sheka, D. D.; Gaididei, Y.; Schäfer, R.; Schmidt, O. G. Nano Lett. 2012, 12, 3961.

(15) Streubel, R.; Kravchuk, V. P.; Sheka, D. D.; Makarov, D.; Kronast, F.; Schmidt, O. G.; Gaididei, Y. Appl. Phys. Lett. 2012, 101, 132419.

(16) Hertel, R.; Kirschner, J. J. Magn. Magn. Mater. 2003, 278, L291.

(17) Landeros, P.; Suarez, O. J.; Cuchillo, A.; Vargas, P. Phys. Rev. B 2009, 79, 024404.

(18) Yan, M.; Andreas, C.; Kákay, A.; García-Sánchez, F.; Hertel, R. Appl. Phys. Lett. 2012, 100, 252401.

(19) Wang, Z. K.; Lim, H. S.; Liu, H. Y.; Ng, S. C.; Kuok, M. H. Phys. Rev. Lett. 2005, 94, 137208.
(20) Escrig, J.; Landeros, P.; Altbir, D.; Vogel, E. E.; Vargas, P. J. Magn. Magn. Mater. 2007, 308, 233.

(21) Escrig, J.; Landeros, P.; Altbir, D.; Vogel, E. E. J. Magn. Magn. Mater. 2007, 310, 2448q.

(22) Castaño, F. J.; Ross, C. A.; Frandsen, C.; Eilez, A.; Gil, D.; Smith, H. I.; Redjdal, M.; Humphrey, F. B. Phys. Rev. Lett. 2003, 67, 184425.

(23) Rüffer, D.; Huber, R.; Berberich, P.; Russo-Averchi, E.; Heiss, M.; Arbiol, J.; Fontcuberta i Morral, A.; Grundler, D. Nanoscale 2012, 4, 4989.

(24) Stipe, B. C.; Mamin, H. J.; Stowe, T. D.; Kenny, T. W.; Rugar, D. Phys. Rev. Lett. 2001, 86, 2874.

(25) Bachmann, J.; Jing, J.; Knez, M.; Barth, S.; Shen, H.; Mathur, S.; Gösele, U.; Nielsch, K. J. Am. Chem. Soc. 2007, 129, 9554.

(26) Daub, M.; Knez, M.; Gösele, U.; Nielsch, K. J. Appl. Phys. 2007, 101, 09J111.

(27) Bachmann, J.; Escrig, J.; Pitzschel, K.; Moreno, J. M. M.; Jing, J.; Görlitz, D.; Altbir, D.; Nielsch, K. J. Appl. Phys. 2009, 105, 07B521.

(28) Rudolph, A.; Soda, M.; Kiessling, M.; Wojtowicz, T.; Schuh, D.; Wegscheider, W.; Zweck, J.; Back, C.; Reiger, E. Nano Lett. 2009, 9, 3860.

(29) Chong, Y. T.; Görlitz, D.; Martens, S.; Yau, M. Y. E.; Allende, S.; Bachmann, J.; Nielsch, K. Adv. Mater. 2010, 22, 2435.

(30) Albrecht, O.; Zierold, R; Allende, S.; Escrig, J.; Patzig, C.; Rauschenbach, B.; Nielsch, K.; Görlitz, D. J. Appl. Phys. 2011, 109, 093910.

(31) Escrig, J.; Bachmann, J.; Jing, J.; Daub, M.; Altbir, D.; Nielsch, K. Phys. Rev. B 2008, 77, 214421.

(32) Bleszynski-Jayich, A. C.; Shanks, W. E.; Peaudecerf, B.; Ginossar, E.; von Oppen, F.; Glazman, L.; Harris, J. G. E. Science 2009, 326, 272.

(33) Jang, J.; Ferguson, D. G.; Vakaryuk, V.; Budakian, R.; Chung, S. B.; Goldbart, P. M.; Maeno, Y. Science 2011, 331, 186.

(34) Banerjee, P.; Wolny, F.; Pelekhov, D. V.; Herman, M. R.; Fong, K. C.; Weissker, U.; Mühl, T.; Obukhov, Yu.; Leonhardt, A.; Büchner, B.; Hammel, P. C. Appl. Phys. Lett. 2010, 96, 252505.

(35) Lee, S.; Moore, E. W.; Hickman, S. A.; Longenecker, J. G.; Marohn, J. A. J. Appl. Phys. 2012, 111, 083911.

(36) Chui, B. W.; Hishinuma, Y.; Budakian, R.; Mamin, H. J.; Kenny, T. W.; Rugar, D. Transducers, 12th Int. Conf. Solid-State Sensors, Actuators Microsyst. 2003, 2, 1120.

(37) Rugar, D.; Mamin, H. J.; Guethner, P. Appl. Phys. Lett. 1989, 55, 2588.

(38) Xue, F.; Peddibhotla, P.; Montinaro, M.; Weber, D. P.; Poggio, M. Appl. Phys. Lett. 2011, 98, 163103.

(39) Stoner, E. C.; Wohlfarth, E. P. Philos. Trans. R. Soc. London, Ser. A 1948, 240, 599.

(40) Kittel, C. Introduction to Solid-State Physics, 8th ed.; Wiley: New York, 2005.

(41) Li, S. P.; Peyrade, D.; Natali, M.; Lebib, A.; Chen, Y.; Ebels, U.; Buda, L. D.; Ounadjela, K. Phys. Rev. Lett. 2001, 86, 1102.

\section{NOTE ADDED AFTER ASAP PUBLICATION}

This paper was published ASAP on November 9, 2012. J. Arbiol has been added as a contributing author. The revised version posted on November 16, 2012. 